Rubrikherausgeber:

Tom Schaberg, Rotenburg (Koordination)

Roland Buhl, Mainz

Adrian Gillissen, Leipzig
Karl Häußinger, Gauting

Rolf Merget, Bochum

Claus Vogelmeier, Marburg

T. O. F. Wagner, Frankfurt
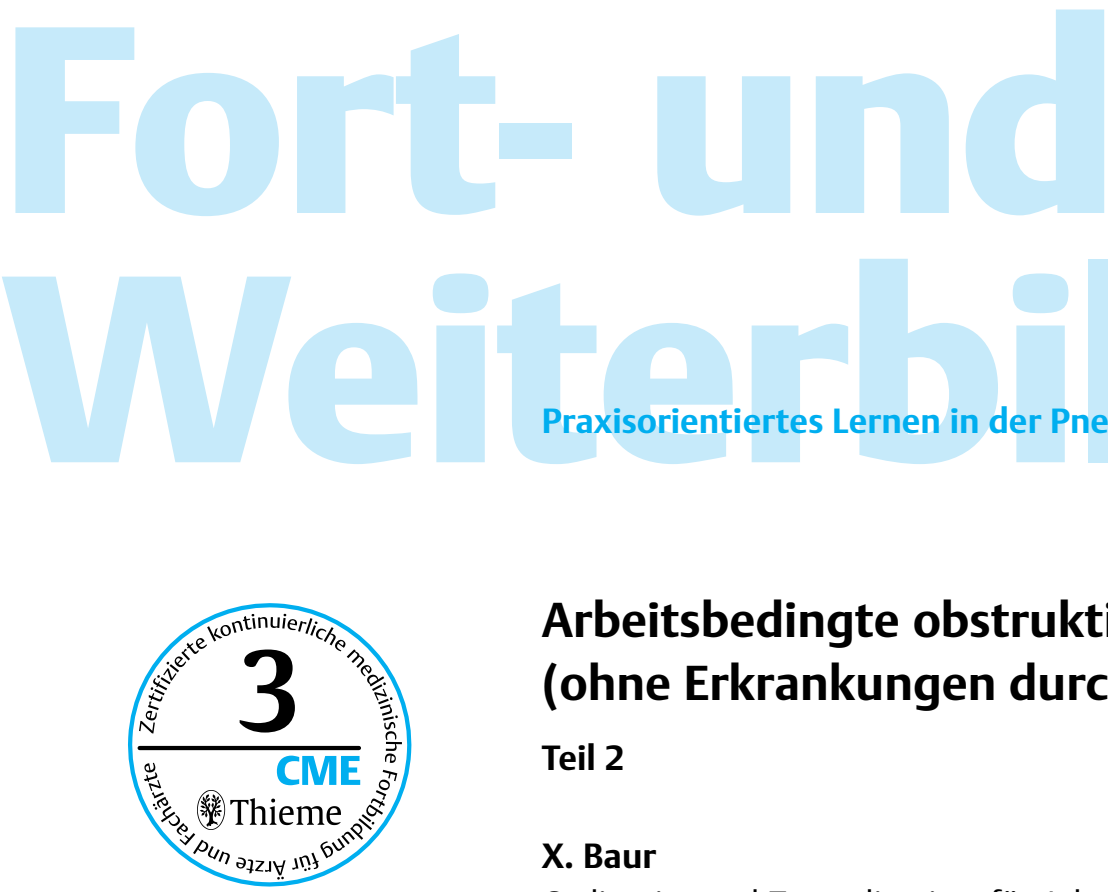

\title{
Arbeitsbedingte obstruktive Atemwegserkrankungen (ohne Erkrankungen durch anorganische Stäube)
}

\section{Teil 2}

\section{Baur}

Ordinariat und Zentralinstitut für Arbeitsmedizin Hamburg

\section{Weiterbildungsziele}

In diesem Beitrag sollen folgende Weiterbildungsziele vermittelt werden:

1. Neuere diagnostische Verfahren

1.1 Atemkondensat-Analyse

1.2 Induziertes Sputum

1.3 NO-Messung

1.4 Beurteilung und Normwerte
2. Differenzialdiagnostische Aspekte

3. Prävention

4. Therapie und Management

5. Prognose und Berufsberatung

6. Berufskrankheitenverfahren 
Neuere diagnostische Verfahren umfassen Analysen des Atemkondensats, des indizierten Sputums und der FeNO.

Für die Atemkondensat-Analyse stehen erstmalig Referenzwerte zur Verfügung.

Die Eosinophilenzahl im induzierten Sputum eignet sich zum Monitoring des arbeitsbedingten Asthmas.

\section{Neuere diagnostische Verfahren}

\subsection{Atemkondensat-Analyse}

Mittels des Gerätes ECOScreen (Fa. Viasys) können nach vorheriger Mundspülung auf nichtinvasive einfache Weise das Atemkondensat der Ausatemluft gesammelt und darin befindliche Komponenten quantitativ bestimmt werden. Dabei wird davon ausgegangen, dass die erfassten Aerosoltröpfchen die Zusammensetzung des extrazellulären bronchoalveolären Flüssigkeitsfilms widerspiegeln.

Das Verfahren wird derzeit noch vorwiegend im Rahmen wissenschaftlicher Untersuchungen eingesetzt. Die Analysen eignen sich auch zur Verlaufskontrolle des arbeitsbedingten Asthmas und erlauben differenzialdiagnostische Aussagen. Eine Festlegung zur Standardisierung ist kürzlich erfolgt [1].

Asthmatiker weisen erhöhte Konzentrationen an $\mathrm{H}_{2} \mathrm{O}_{2}$, NO-Metaboliten, IL-4, IL-5, 8-iso-Prostan, LTE4, LTB4, erniedrigte Werte von IFN- $\gamma$ und des pH sowie ein verändertes Proteinspektrum auf.

Referenzwerte (laborabhängig): $\mathrm{pH} 7,51-7,67, \mathrm{H}_{2} \mathrm{O}_{2} 0,08-0,41 \mathrm{mM}$, LTB4 5,8-281 pg/ $\mathrm{ml}$, LTE4 $11,4-14,6 \mathrm{pg} / \mathrm{ml}$, IL-4 $29,5-41,9 \mathrm{pg} / \mathrm{ml}$, IL-6 $7,69-7,91 \mathrm{pg} / \mathrm{ml}$, IFN- $\gamma$ $4,7-5,5 \mathrm{pg} / \mathrm{ml}, \mathrm{IL}-5<17 \mathrm{pg} / \mathrm{ml}, 8$-iso-Prostan $1,5-6,6 \mathrm{pg} / \mathrm{ml}$.

Studien zur diagnostischen Wertigkeit für arbeitsbedingte obstruktive Atemwegserkrankungen stehen noch aus.

\subsection{Induziertes Sputum}

Durch Inhalation hypertoner Kochsalzlösung lässt sich induziertes Sputum gewinnen [2]. Mehrere Untersuchungen belegen, dass sich v. a. die Eosinophilenzahl zum Monitoring des arbeitsbedingten Asthmas eignet. So fanden Obata u. Mitarb. [3] 6 und 24 Stunden nach Provokation mit Plicatsäure aus Zedernholz unter Respondern einen Eosinophilenanstieg, der sich umgekehrt proportional zum $\mathrm{FEV}_{1}$-Abfall verhielt.

Kürzlich beschrieben Lemiere u. Mitarb. [4] im induzierten Sputum von Patienten mit Isocyanat-Asthma nach entsprechender inhalativer Exposition eine verstärkte Expression der Leukotrien-Rezeptoren CysLLT1 und BLT1 auf Neutrophilen, ferner einen Anstieg von Leukotrien LTB 4, IL-8 und eine Neutrophilie.

Auch Getreidestaub induziert eine Zunahme von IL-8 und Neutrophilen [5].

In einer prospektiven Multizenter-Studie untersuchten Girand u. Mitarb. [6] 49 Patienten, davon 23 mit positivem Allergen-Provokationstest, nach einer zweiwöchigen Arbeitsphase und nach einer zweiwöchigen Arbeitspause. Dabei kamen ein PEF-Monitoring und die Quantifizierung der Eosinophilen im induzierten Sputum zur Anwendung. Die computerisierte PEF-Analyse war der individuellen visuellen Auswertung deutlich unterlegen. Die zusätzliche Sputum-Untersuchung erhöhte die Spezifität des PEF-Monitorings um 18\% (bei Anstieg der Eosinophilen $\geq 1 \%$ ). Gleichzeitig nahm die Sensitivität um 8,2\% zu. Die Studie belegt - ähnlich wie frühere Arbeiten während und außerhalb von Arbeitsphasen bzw. nach arbeitsplatzbezogenen spezifischen Expositionstesten - die diagnostische Wertigkeit der Sputum-Untersuchung für das arbeitsbedingte Asthma.

\subsection{NO-Messung}

Die Chemolumeniszenz-Analyse erlaubt unter standardisierten Bedingungen (geschlossenes Velum, weitgehend konstanter Atemfluss von $50 \mathrm{~mL} / \mathrm{s}$, Widerstand von ca. 10 mbar), die Messungen der aus dem Bronchoalveolarraum stammenden, exhalier- 
Die Messung des aus den tiefen Atemwegen exhalierten NO ist eine viel versprechende Methode zur Erfassung der inflammatorischen Reaktion in der bronchialen Mukosa. ten NO-Fraktion (FeNO). Eine Modifikation des NO, die im Gewebe nachweisbar ist, stellt das Proteinaddukt 3-Nitrothyrosin dar.

Ein FeNO-Anstieg ca. 1 Tag nach einer Allergenbelastung, z. B. mit Isocyanaten oder Latex, ist ein guter Indikator für eine allergische Atemwegsreaktion und -entzündung [7] (Abb. 1).

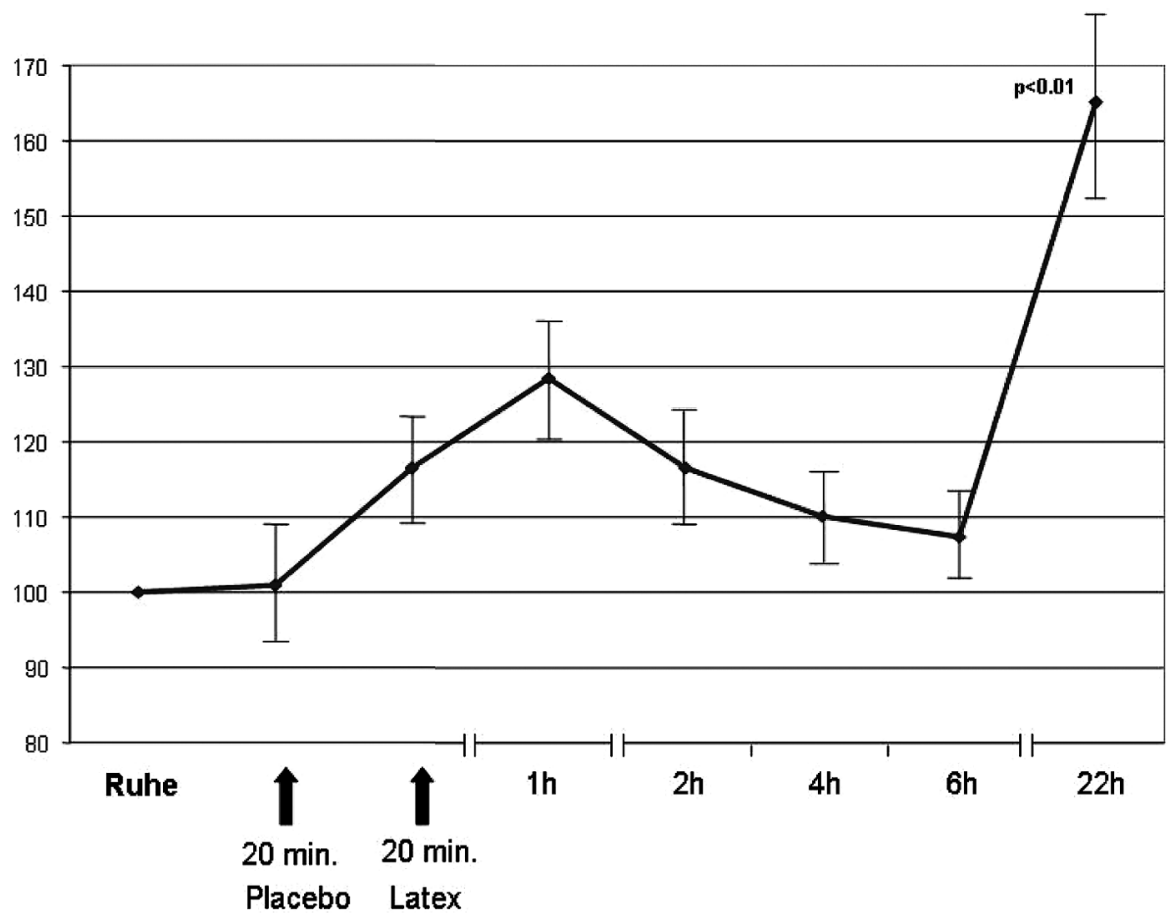

Abb. 1 Signifikanter FeNO-Anstieg $22 \mathrm{~h}$ nach arbeitsbezogener Latexallergen-Exposition (Mittelwert \pm SEM; $n=31$ ).

\subsection{Beurteilung und Normwerte}

- Normbereich des FeNO: 6-9,4 ppb (laborabhängig).

- Raucher haben erniedrigte FeNO-Werte.

- Patienten mit Atemwegsallergien haben ein erhöhtes FeNO.

- Die Allergenexposition führt beim Sensibilisierten nach etwa einem Tag zu einem 50- bis $100 \%$ igen Anstieg des FeNO.

- Die erhöhten Werte der Asthmatiker und der Methacholin-Hyperreagiblen normalisieren sich unter inhalativer Glukokortikoid-Therapie (Ausnahme: steroidresistente Patienten).

- Die nasale NO-Konzentration ist ca. 100fach höher als FeNO.

- Patienten mit primärer ziliärer Dyskinesie (z. B. Karthagener-Syndrom) haben eine stark erniedrigte nasale und bronchiale NO-Konzentration.

\section{Differenzialdiagnostische Aspekte}

Zunächst sind durch Berufsallergene hervorgerufene allergische obstruktive Atemwegserkrankungen (BK-Nr. 4301, in Deutschland ca. 1000 anerkannte Fälle/Jahr) von solchen, die durch chemisch-irritative oder toxisch wirkende Stoffe verursacht werden, abzugrenzen (BK 4302, ca. 215 anerkannte Fälle/Jahr). Das Isocyanat-Asthma und die seltene Isocyanat-bedingte Alveolitis sind separat aufzuführen (BK-Nr. 1315, ca. 50 Fälle/Jahr). 
Auch nicht durch die Arbeit bedingte pulmonale Erkrankungen gehören zur Differenzialdiagnose.
Primärprävention hat Vorrang vor der Sekundärprävention.
Tab. 1 Wichtige Differenzialdiagnosen arbeitsbedingter obstruktiver Atemwegserkrankungen

\begin{tabular}{|c|c|}
\hline Krankheitsbild & Charakteristika \\
\hline Intrinsic Asthma & unabhängig von exogenen Faktoren, häufig Infekt-exazerbiert \\
\hline $\begin{array}{l}\text { Asthma bronchiale durch ubiquitäre } \\
\text { Allergene }\end{array}$ & expositionsabhängiges Asthma \\
\hline COPD anderer Genese & Husten, Auswurf, Belastungsdyspnoe, progredienter Verlauf \\
\hline exogen allergische Alveolitis & $\begin{array}{l}\text { organische Aerosole, Symptome nach mehrstündiger Latenz- } \\
\text { zeit, fleckige Infiltrate, Lungenfibrose }\end{array}$ \\
\hline ODTS & $\begin{array}{l}\text { grippeartige Beschwerden, oft auch erschwerte Atmung nach } \\
\text { Belastung mit Endotoxin-haltigen Stäuben }\end{array}$ \\
\hline Metallrauchfieber & $\begin{array}{l}\text { Fieber, Beklemmungsgefühl einige Stunden nach Exposition } \\
\text { gegenüber v. a. Zinkoxid-haltigem Rauch }\end{array}$ \\
\hline toxisches Lungenödem & $\begin{array}{l}\text { Exposition gegenüber chemischen Noxen, mehrstündige La- } \\
\text { tenzzeit, Infiltrate }\end{array}$ \\
\hline $\begin{array}{l}\text { allergische bronchopulmonale } \\
\text { Aspergillose (ABPA) }\end{array}$ & $\begin{array}{l}\text { Asthma, Infiltrate, Aspergillus-spezifisches } \lg E+\lg G, \lg E \uparrow \text {, } \\
\text { zentr. Bronchiektasen, Aspergillennachweis }\end{array}$ \\
\hline pulmonale Vaskulitis & Infiltrate, Eosinophilie, histologisch: Vaskulitis \\
\hline Lungenemphysem & chronischer Verlauf, Emphysemzeichen \\
\hline zystische Fibrose & $\begin{array}{l}\text { exokrine Pankreasinsuffizienz, Kachexie, zähes Sputum, patho- } \\
\text { logischer Schweißtest, Bullae }\end{array}$ \\
\hline Bronchiolitis obliterans & $\begin{array}{l}\text { polyphonische RG, Überblähung und fokale Atelektasen, vor } \\
\text { allem bei Graft-versus-Host-Reaktionen }\end{array}$ \\
\hline Pneumokoniosen & Lungenschatten im Röntgenbild, Exposition \\
\hline chronische Bronchitis & Sputum $\uparrow$, Husten $\uparrow$ \\
\hline Bronchialkarzinom, Polyp & langsamer Beginn, Stridor \\
\hline Fremdkörperaspiration & Verschlucken, meist plötzlicher Beginn der Beschwerden \\
\hline Asthma cardiale & Herzinsuffizienz, Orthopnoe, feuchte RG, Herzgröße \\
\hline Trachealstenose & Inspiratorischer Stridor; Struma, Z.n. Langzeit-Intubation \\
\hline Tracheomalazie & Langzeit-Intubation \\
\hline okklusives Schlafapnoe-Syndrom & $\begin{array}{l}\text { Apnoephasen, lautes Schnarchen; neuromuskuläre Störung der } \\
\text { Zungengrund- u. Pharynx-Muskulatur }\end{array}$ \\
\hline Rekurrensparese & Tumor, Zustand nach Strumaresektion \\
\hline Pseudokrupp & nachts bellender Husten, Stridor \\
\hline Angioödem & Allergie oder C1-Esterase-Inhibitor-Mangel \\
\hline Hyperventilationssyndrom & Parästhesien, karpopedale Spasmen, Angstzustände \\
\hline Vocal cord dysfunction syndrome & $\begin{array}{l}\text { paradoxe Adduktion der Stimmbänder während Inspiration; } \\
\text { psychische Komponente; oft sind jüngere Frauen betroffen }\end{array}$ \\
\hline
\end{tabular}

Tab. 1 fasst wichtige Differenzialdiagnosen arbeitsbedingter obstruktiver Atemwegserkrankungen zusammen. Im Vordergrund stehen das Asthma bronchiale und die COPD anderer Ursachen sowie durch spezielle Expositionen am Arbeitsplatz ausgelöste sonstige Erkrankungen, die nicht selten schwierig abzugrenzen sind. Beispiele sind: ODTS, Metallrauchfieber, toxisches Lungenödem, Silikose, Asbestose, andere Pneumokoniosen, exogen allergische Alveolitiden wie die Farmer- und Vogelhalterlunge.

\section{Prävention}

Der Primärprävention (Meidung oder zumindest Minimierung der Exposition) kommt eine vorrangige Bedeutung zu. Medizinische Überwachungsprogramme in Betrieben mit regelmäßigen Vorsorgeuntersuchungen von Risikogruppen (vgl. Tab. 2-4) verhindern infolge früher Diagnosestellung und Intervention Erkrankungen bzw. deren Fortschreiten (Sekundärprävention). Nach internationaler Übereinstimmung sollten gefährdete Arbeitnehmer jährlich einen evaluierten Anamnesefragebogen ausfüllen, in dem sie ihre beruflichen Belastungen, ihren Gesundheitszustand und arbeitsassoziierte Beschwerden angeben [8-10]. Tarlo u. Mitarb. [11] und Tarlo und Liss [12] konnten 
Welche Vorsorgeuntersuchungen müssen im Rahmen der Arbeitsmedizin bei obstruktiven Atemwegserkrankungen erfolgen?

Tätigkeiten, bei denen arbeitsmedizinische Vorsorgeuntersuchungen vorgeschrieben sind.

Tätigkeiten, bei denen arbeitsmedizinische Vorsorgeuntersuchungen empfehlenswert sind.
Tab. 2 Arbeitsmedizinische Vorsorgeuntersuchungen für obstruktive Atemwegserkrankungen $\left(\mathrm{G} 23^{*}\right)[18]$

- detaillierte klinische und Arbeits-Anamnese

- körperlicher Status

- Lungenfunktionsprüfung (Spirometrie ausschließlich der Fluss-Volumen-Kurve; bei Indikation ergänzt durch Bodyplethysmographie und Untersuchung auf bronchiale Hyperreagibilität wie MethacholinProvokationstest)

- ggf. Allergiediagnostik

- eingehende, individuelle Beratung auf Basis der Untersuchungsergebnisse, ggf. Einleitung eines Antiraucherprogramms

*Pflicht bei den in Tab. 3 aufgeführten Expositionen und Tätigkeiten anzubieten und empfohlen für Tätigkeiten entsprechend Tab. 4.

Tab. 3 Tätigkeiten, bei denen arbeitsmedizinische Vorsorgeuntersuchungen entsprechend dem Berufsgenossenschaftlichen Grundsatz G 23 zu veranlassen sind [19] (siehe auch zusätzlich Liste der Stoffe in Anhang V Nr. 1 der GefstoffV)

- Schweißen und Trennen von Metallen bei Überschreitung eines Luftgrenzwertes von $3 \mathrm{mg} / \mathrm{m}^{3}$ Gesamtschweißrauch

- Tätigkeiten mit Belastungen durch Getreide- und Futtermittelstäube bei Überschreitung eines Luftgrenzwertes von $4 \mathrm{mg} / \mathrm{m}^{3}$ einatembaren Staubs

- Tätigkeiten mit Belastungen durch Isocyanate, bei denen ein regelmäßiger Hautkontakt nicht vermieden werden kann oder ein Luftgrenzwert von $0,05 \mathrm{mg} / \mathrm{m}^{3}$ überschritten ist

- Tätigkeiten mit Belastungen durch Labortierstaub in Tierhaltungsräumen und -anlagen

- Abbruch-, Sanierungs- und Instandhaltungsarbeiten mit Asbest bei Überschreitung einer Asbestfaserkonzentration von 15000 Fasern/m³

- Tätigkeiten mit Benutzung von Naturgummi-Latexhandschuhen mit mehr als $30 \mu \mathrm{g}$ Protein/g Handschuh

- Tätigkeiten mit Belastungen durch unausgehärtete Expoxidharze und Kontakt über die Haut oder die Atemwege

- alveolengängige Staubfraktion $>3 \mathrm{mg} / \mathrm{m}^{3}$

- einatembare Staubfraktion $>10 \mathrm{mg} / \mathrm{m}^{3}$

Tab. 4 Tätigkeiten, bei denen arbeitsmedizinische Vorsorgeuntersuchungen entsprechend G 23, z. T. auch darüber hinaus, anzubieten sind [19]

- Begasungen nach Anhang III Nr. 5

- Schädlingsbekämpfung nach Anhang III Nr. 4

- Tätigkeiten mit organischen Lösungsmittelgemischen aus den folgenden Stoffen: n-Hexan, n-Heptan, 2-Butanon, 2-Hexanon, Methanol, Ethanol, 2-Methoxyethanol, Benzol, Toluol, Xylol, Styrol, Dichlormethan, 1,1,1-Trichlorethan, Trichlorethan, Tetrachlorethen

- Schweißen und Trennen von Metallen bei Einhaltung eines Luftgrenzwertes von $3 \mathrm{mg} / \mathrm{m}^{3}$

- Tätigkeiten mit Belastung durch Getreide- und Futtermittelstäube bei Überschreitung eines Luftgrenzwertes von $1 \mathrm{mg} / \mathrm{m}^{3}$ einatembaren Staubs

für Isocyanat-Arbeiter und Merget u. Mitarb. [13] für Platin-exponierte Arbeitnehmer den Nutzen arbeitsmedizinischer Vorsorgeuntersuchungen nachweisen. In einer 10-jährigen arbeitsmedizinischen Überwachung belegte Smith [14] eine Abnahme neuer Asthmaerkrankungen auf etwa 25\% des Ausgangswerts. Eine Verlaufsuntersuchung von Studierenden der Zahnmedizin über 5 Jahre ergab, dass nach Umstellung auf Vinyl-Handschuhe keine neuen Latex-Sensibilisierungen mehr auftraten [15].

Interventionen und Verlaufsuntersuchungen in Krankenhäusern mit einem gehäuften Auftreten von Latexallergien (Ersatz allergenreicher gepuderter Handschuhe durch 
Die Kosten-Nutzen-Analyse der arbeitsmedizinischen Vorsorgeuntersuchungen ist positiv zu bewerten.

Tertiärprävention entspricht rehabilitativen Maßnahmen.
Handschuhe mit geringem Allergengehalt, zum Teil ohne Puder) zeigen eine signifikante Abnahme von Neuerkrankungen $[16,17]$.

Die arbeitsmedizinische Vorsorgeuntersuchung G 23 wird außerdem regelmäßig in zwei- bis dreijährigen Abständen für alle Risikopatienten (z.B. vorbestehende Atemwegserkrankungen oder Heuschnupfen) und Berufsgruppen mit stark erhöhtem Erkrankungsrisiko (z.B. Bäcker, Reinigungspersonal, Chemiearbeiter) empfohlen.

Aktuelle sozialpolitische Diskussionen befassen sich jenseits von ethischen Aspekten mit Kosten-Nutzen-Relationen arbeitsmedizinischer Vorsorgeuntersuchungen und Überwachungsprogramme. Kürzlich berechneten Wild u. Mitarb. [8] an einem Modell für Isocyanat-Arbeiter einen volkswirtschaftlichen Nutzen derselben, wobei Kosten zu Lasten des Unternehmers in Höhe von \$24000 pro qualitätsadjustiertes Lebensjahr entstanden ( $\$ 13,33$ pro symptomfreien Tag, \$64000 pro verhinderten Erwerbsunfähigkeitsfall). Insgesamt ließen sich demnach durch die Vorsorgeuntersuchung innerhalb von 10 Jahren unter 100000 Isocyanat-Arbeitern 683 Erwerbsunfähigkeitsfälle verhindern. Dabei sind, wie in einem Kommentar von LaMontagne [20] zu recht ausgeführt wird, der gesamte volkswirtschaftliche Nutzen weit höher zu veranschlagen und der Rechtsanspruch auf einen „gesunden Arbeitsplatz“ zu berücksichtigen.

Der Verlust des Arbeitsplatzes ist mit erheblichen Nachteilen und selbst bei anerkannter Berufskrankheit oft mit Einkommenseinbußen und anderen Nachteilen assoziiert. Unter Berücksichtigung des Wunsches der/des Erkrankten und der häufig schwierigen Arbeitsmarktsituation sollte versucht werden, durch organisatorische und andere sekundarpräventive Maßnahmen den Arbeitsplatz zu erhalten.

Die Tertiärprävention (medizinische Rehabilitation) zielt auf die Wiederherstellung der Gesundheit oder zumindest die Verhinderung der Chronifizierung der obstruktiven Atemwegserkrankungen. Hierzu zählen die medikamentöse Therapie (unterscheidet sich nicht von jener, die bei nicht-berufsbedingten Erkrankungen angewendet wird), spezielle Schutzmaßnahmen am Arbeitsplatz, in schweren Krankheitsfällen auch Heilbehandlungen (Kuraufenthalte in entsprechend spezialisierten Rehabilitationszentren).

\section{Therapie und Management}

Ein Arbeitsplatzwechsel ist indiziert, wenn primärpräventive und organisatorische Maßnahmen nicht ausreichen.
Die Prognose arbeitsbedingter obstruktiver Atemwegserkrankungen verschlechtert sich durch fortgesetzte ursächliche Exposition des Erkrankten. Der Arbeitsplatzwechsel ist daher die wichtigste Maßnahme, wenn primärpräventiv und durch organisatorische Maßnahmen am Arbeitsplatz nicht Karenz eingehalten werden kann. Wird die Exposition dennoch versuchsweise aufrechterhalten, sollte unbedingt eine engmaschige Begleitung durch den Betriebsarzt und Pneumologen erfolgen sowie konsequent adäquat Atemschutz (Feinstaubfilter, FPP 2) getragen werden. Letzterer eignet sich gut präventiv für Gesunde, bei bereits bestehender Symptomatik ist er nur noch von begrenzter Wirksamkeit [21,22].

Da Rauchen für sich alleine schon zu einer Verschlechterung der Lungenfunktion führt und auch bestimmte Sensibilisierungen (Platinsalze, Säureanhydride, Labortiere) begünstigt, sollten in den Betrieben neben der individuellen Beratung auch Antiraucherkampagnen initiiert werden.

Wichtig sind darüber hinaus Patientenschulungen mit besonderer Betonung von Strategien zur Vermeidung arbeitsbedingter Trigger, der Erkennung von Exazerbationen und der Symptom-orientierten Optimierung der medikamentösen Therapie. Letztere 
Es ist nicht vorhersagbar, wer eine obstruktive Atemwegserkrankung entwickelt.

Genetische Risikofaktoren spielen bei arbeitsbedingten obstruktiven Atemwegserkrankungen eine wichtige Rolle.
Jeder Arzt muss den begründeten Verdacht auf eine Berufskrankheit dem Unfallversicherungsträger oder dem staatlichen Gewerbearzt melden. unterscheidet sich nicht von Atemwegserkrankungen, die nicht auf arbeitsbedingte Ursachen zurückgehen.

Eine Hyposensibilisierung gegen Berufsallergene wird nicht empfohlen, zumal der Erfolg einer solchen Behandlung in keiner kontrollierten Studie belegt ist.

\section{Prognose und Berufsberatung}

Die Vorhersage, wer eine arbeitsbedingte obstruktive Atemwegserkrankung entwickelt, ist nicht möglich. Der prädiktive Wert bekannter genetischer Marker (Tab.5) und einer bronchialen Überempfindlichkeit ist bisher nicht untersucht und sicher gering, so dass - mit Ausnahme von ganz spezieller Situationen (z.B. vorbestehendes schweres Asthmaleiden, höhergradige Sensibilisierung auf Berufsallergene) - Personen nicht von der Ausübung bestimmter Berufe ausgeschlossen werden sollen. Ein erhöhtes Erkrankungsrisiko liegt bei Vorbestehen einer Sensibilisierung auf Katzen und andere Haustiere, einer bronchialen Hyperreagibilität und einer eingeschränkten $\mathrm{FEV}_{1}$ vor, wenn eine Tätigkeit als Tierpfleger aufgenommen wird [23]. Es gibt auch Hinweise, dass Patienten mit Graspollensensibilisierung infolge kreuzreagierender Allergene ein erhöhtes Risiko für eine Mehlstauballergie haben.

Tab. 5 Prädisponierende genetische Faktoren für arbeitsbedingte obstruktive Atemwegserkrankungen [24]

\begin{tabular}{|ll}
\hline Exposition & Gene \\
\hline & \\
\hline Isocyanate & GSTP1, GSTM1, GSTM3, GSTT1, HLAll-DQA1, HLAll-DQB1, \\
& HLAll-DRB1,2,3,4, HLA-Klasse 1 \\
& TNF \\
& TCR V $\beta$ \\
& AAT \\
& NAT1 y NAT2 \\
& ADRB2 \\
& hochaffiner IgE-Rezeptor \\
& TNF-308 \\
\hline Aluminium-Produktion & AAT \\
& HLAI-Allotypen der Ig \\
& HLAll-DP, HLAll-DQ5, HLAll-DR1 \\
& TLR2, TLR4 \\
& AAT \\
\hline Säureanhydride & HLAll-DRB1, HLALL-DQB1 \\
\hline Landwirtschaft & HLAll-DQB1, HLAll-DRB1, HLAll-A, HLAll-B, HLAll-C \\
& HLAll-DRB, HLAll-DPB, HLAll-DQA, HLAll-DQB \\
\hline Holzstäube & \\
\hline Labortiere & \\
\hline Platinsalz-Komplexe & \\
\hline
\end{tabular}

Auf diese Zusammenhänge ist im Rahmen der Berufswahl, der Untersuchung nach dem Jugendschutzgesetz sowie der Einstellungsuntersuchung vor Aufnahme einer neuen Beschäftigung hinzuweisen. Letztere dient außerdem der Dokumentation des initialen Gesundheitszustandes.

\section{Berufskrankheitenverfahren}

Jeder Arzt und Zahnarzt ist gemäß §202 SGB VII verpflichtet, den begründeten Verdacht auf eine Berufskrankheit dem Unfallversicherungsträgern (Berufsgenossenschaft) oder der für den medizinischen Arbeitsschutz zuständigen staatlichen Stelle (staatlicher Gewerbearzt, Landesgewerbearzt) mitzuteilen. Nach Ermittlung der Zuständigkeit, des Arbeitsverhältnisses und der individuellen Belastung veranlasst die 
Tab. 6 Einschätzung des medizinischen funktionellen Teils der Minderung der Erwerbsfähigkeit (MdE) bei Atemwegs- und Lungenkrankheiten $[25,26]$.

Zu beachten ist, dass der MdE-Wert anzunehmen ist, für den die Mehrheit der einzelnen Angaben/Befunde spricht; so rechtfertigt z. B. die tägliche inhalative Kortikoid-Medikation allein keine MdE von 40 oder mehr Prozent

\begin{tabular}{|c|c|c|c|c|c|c|c|}
\hline MdE \% & Anamnese & Klinik & $\begin{array}{l}\text { Lungenfunktion } \\
\text { (Spirometrie, Body- } \\
\text { plethysmographie)* }\end{array}$ & $\begin{array}{l}\text { Belastungs- } \\
\text { untersuchung mit } \\
\text { Blutgasbestimmung }\end{array}$ & Spiroergometrie & Therapie & MdE \% \\
\hline 10 & $\begin{array}{l}\text { geringe Beschwerden; } \\
\text { unter Therapie keine } \\
\text { Beschwerden }\end{array}$ & Normalbefund & $\begin{array}{l}\text { Grenzbereich; subklinische } \\
\text { Hyperreagibilität }\end{array}$ & Normoxämie & $\begin{array}{l}\text { Insuffizienz- } \\
\text { kriterien** bei } \\
\text { hoher Belastung }\end{array}$ & $\begin{array}{l}\text { keine oder gele- } \\
\text { gentlich Broncho- } \\
\text { dilatatoren u./o. } \\
\text { Antihistaminika }\end{array}$ & 10 \\
\hline 20 & $\begin{array}{l}\text { keine völlige Be- } \\
\text { schwerdefreiheit unter } \\
\text { Therapie. Leichtgradige }\end{array}$ & $\begin{array}{l}\text { Giemen unter- } \\
\text { schiedlichen } \\
\text { Grades }\end{array}$ & $\begin{array}{l}\text { leichtgradige Veränderungen } \\
\text { überwiegen; reversible Bron- } \\
\text { chialobstruktion: VC, } T_{L, c 0}\end{array}$ & $\begin{array}{l}\text { Normoxämie oder } \\
\text { Hypoxämie bei hoher } \\
\text { Belastung }\end{array}$ & & & 20 \\
\hline $\begin{array}{l}30 \\
40\end{array}$ & $\begin{array}{l}\text { Belastungsdyspnoe. } \\
\text { Periodisch auftretende } \\
\text { Asthma-Anfälle }\end{array}$ & & $\begin{array}{l}\mathrm{FEV}_{1} 99-75 \% \\
\mathrm{R}_{\mathrm{t}} 0,31-0,49 \\
\text { RV, IGV } 101-120 \% \\
\text { o. } 99-80 \%\end{array}$ & $\begin{array}{l}\text { Hypoxämie bei hoher } \\
\text { Belastung }\end{array}$ & $\begin{array}{l}\text { Insuffizienz- } \\
\text { kriterien** bei } \\
\text { mittlerer } \\
\text { Belastung }\end{array}$ & $\begin{array}{l}\text { täglich inhalative } \\
\text { Kortikoide und } \\
\text { Bronchodilatoren }\end{array}$ & 30 \\
\hline 50 & $\begin{array}{l}\text { mittelgradige Belas- } \\
\text { tungsdyspnoe } \\
\text { (z. B. Pause nach 2-3 } \\
\text { Stockwerken). Tägliche } \\
\text { Atembeschwerden. } \\
\text { Geringe nächtliche } \\
\text { Beschwerden }\end{array}$ & $\begin{array}{l}\text { Cor pulmonale } \\
\text { ohne Insuffi- } \\
\text { zienzzeichen }\end{array}$ & $\begin{array}{l}\text { mittelgradige Veränderungen } \\
\text { überwiegen: } \\
\text { VC, } T_{L, c o}, \mathrm{FEV}_{1} \\
74-50 \% \text {; } \\
\mathrm{R}_{\mathrm{t}} 0,5-0,9 \text {, nicht voll } \\
\text { reversibel; } \\
\text { RV, IGV } 121-145 \% \text { o. } 79-60 \%\end{array}$ & $\begin{array}{l}\text { Hypoxämie bei } \\
\text { mittlerer Belastung }\end{array}$ & & & $\begin{array}{l}50 \\
60\end{array}$ \\
\hline 70 & $\begin{array}{l}\text { hochgradige Belas- } \\
\text { tungsdyspnoe } \\
\text { (z. B. Pause nach } \\
1 \text { Stockwerk). }\end{array}$ & $\begin{array}{l}\text { Cor pulmonale } \\
\text { mit reversiblen } \\
\text { Zeichen der } \\
\text { Rechts-Herz- } \\
\text { Insuffizienz }\end{array}$ & & $\begin{array}{l}\text { Hypoxämie bei } \\
\text { leichter Belastung }\end{array}$ & $\begin{array}{l}\text { Insuffizienz- } \\
\text { kriterien** } \\
\text { bei leichter } \\
\text { Belastung }\end{array}$ & $\begin{array}{l}\text { zusätzlich orale } \\
\text { Kortikoide, sonsti- } \\
\text { ge Medikation } \\
\text { notwendig. } \\
\mathrm{O}_{2} \text {-Therapie }\end{array}$ & 70 \\
\hline 80 & $\begin{array}{l}\text { tägliche Asthmaanfälle. } \\
\text { Regelmäßig nächtliche } \\
\text { Atemnotzustände }\end{array}$ & & $\begin{array}{l}\text { hochgradige Veränderungen } \\
\text { Überwiegen: VC, } T_{L, c o}, F_{1} \\
<50 \% \text {; } R_{t}>0,9 ; R V \text {, IGV }>145 \% \\
\text { o. }<60 \%\end{array}$ & & & & 80 \\
\hline 90 & $\begin{array}{l}\text { Gehstrecke ohne Pause } \\
<100 \text { m oder }<8 \text { Stufen }\end{array}$ & $\begin{array}{l}\text { Ruhedyspnoe, } \\
\text { zeitweise statio- } \\
\text { näre Behandlung. } \\
\text { Polyglobulie. }\end{array}$ & & $\begin{array}{l}\text { Hypoxämie in Ruhe } \\
\text { bei Normokapnie }\end{array}$ & & & 90 \\
\hline 100 & $\begin{array}{l}\text { Ruhedyspnoe (Hilfe } \\
\text { beim Essen u./o. } \\
\text { Kleiden notwendig). } \\
\text { Wiederholt lebens- } \\
\text { bedrohliche Asthma- } \\
\text { anfälle }\end{array}$ & $\begin{array}{l}\text { Cor pulmonale } \\
\text { mit irreversibler } \\
\text { Rechtsherz- } \\
\text { insuffizienz }\end{array}$ & $\begin{array}{l}\text { forcierte Atemmanöver nicht } \\
\text { möglich }\end{array}$ & $\begin{array}{l}\text { Hypoxämie und } \\
\text { Hyperkapnie in Ruhe }\end{array}$ & & $\begin{array}{l}\text { trotz optimaler } \\
\text { Therapie nicht } \\
\text { beherrschbare } \\
\text { Atemwegs- } \\
\text { erkrankung }\end{array}$ & 100 \\
\hline
\end{tabular}

* Abweichungen beziehen sich auf folgende Sollgrenzwerte: $R_{t}\left(R_{\text {eff }}\right)$ nach Matthys u. Mitarb. [27];

IGV nach Islam und Ulmer [28]/Ulmer u. Mitarb. [29]; RV, TLC nach Quanjer u. Mitarb. [30]; VC max $_{\text {, FVC, FEV }}$, Fluss-Volumen-Kurve nach Brändli u. Mitarb. [31]; Blutgase nach Woitowitz u. Mitarb. [32]

** Abweichungen von Normwerttabellen, v. a. von $\mathrm{VO}_{2 \max }, \mathrm{VO}_{2} \mathrm{AT}, \mathrm{P}(\mathrm{A}-\mathrm{a}) \mathrm{O}_{2} \mathrm{AT}, \mathrm{RQ}>1,0$, Laktat $>4 \mathrm{mmol} / \mathrm{l}$.

Probleme der Begutachtung bei arbeitsbedingten obstruktiven Atemwegserkrankungen.
Unfallversicherung in der Regel ein medizinisches Zusammenhangsgutachten. In diesem sind eine Objektivierung des Krankheitsbildes und seines Schweregrades sowie eine ätiologische Klärung bestmöglich vorzunehmen und gegebenenfalls der durch die Berufskrankheit bedingte klinisch-medizinische Teil der Minderung der Erwerbsfähigkeit (MdE) auf dem allgemeinen Arbeitsmarkt abzuschätzen (Tab. 6). Eine zentrale Rolle nimmt dabei die spezielle Kenntnisse voraussetzende Arbeitsanamnese und Bewertung der Belastung ein. Es empfiehlt sich, die Sicherheitsdatenblätter aller verwendeten Arbeitsstoffe eingehend zu prüfen, ebenso den Bericht des technischen Aufsichtsbeamten der Unfallversicherung (TAB-Bericht; falls letzterer fehlt, sollte er unverzüglich angefordert werden).

Bei gleichzeitigem Nachweis von arbeits- und umweltbedingten Sensibilisierungen (z.B. gegen Mehl und Gräserpollen beim Bäcker) oder Noxeneinwirkungen ist zu prü- 
Die Prognose der arbeitsbedingten obstruktiven Atemwegserkrankungen ist ungünstig. fen, ob den arbeitsbedingten Faktoren eine wesentliche Teilursache für die Erkrankung zukommt (mindestens zu einem Drittel). Ist diese Frage zu bejahen, wird bei zeitlich parallelen Einwirkungen der Gesamtschaden als Berufskrankheit eingestuft. Haben arbeitsbedingte Faktoren zu einer richtunggebenden Verschlimmerung einer vorbestehenden obstruktiven Atemwegserkrankung geführt, ist der Verschlimmerungsteil als entschädigungspflichtige Berufskrankheit zu werten.

\section{Schlussbetrachtungen}

Die Diagnostik und korrekte versicherungsrechtliche Zuordnung arbeitsbedingter obstruktiver Atemwegserkrankungen ist aufwändig und schwierig. Dies betrifft insbesondere irritative und toxische Formen, die oft Ausschlussdiagnosen darstellen und pathogenetisch auf kumulativen Schädigungen des Respirationstrakts beruhen. Das heißt, die Diagnose basiert hier v.a. auf dem Zusammenhang zwischen dem Beginn und der Dauer einer erheblichen Belastung einerseits und dem Auftreten, der Progredienz und dem Langzeitverlauf des Krankheitsbildes andererseits bei Fehlen anderer, im Vordergrund stehender Ursachen. Auf das wichtige diagnostische Werkzeug serieller Lungenfunktionsmessungen am Arbeitsplatz, zum Beispiel mittels Peak Expiratory Flow (PEF)-Monitoring, heute besser mittels elektronischer Mini-Spirometer, sei besonders hingewiesen.

Eine Reihe von Untersuchungen belegt, dass die Prognose arbeitsbedingter obstruktiver Atemwegserkrankungen ungünstig ist. Padoan u. Mitarb. [35] fanden unter 87 Isocyanatarbeitern noch 10 Jahre nach Berufsaufgabe überwiegend Einschränkungen der Lungenfunktion. Vandenplas u. Mitarb. [36] weisen auf die negativen sozioökonomischen Auswirkungen arbeitsbedingter Atemwegserkrankungen hin.

\section{Literatur}

1 Horvath I, Hunt J, Barnes PJ. Exhaled breath condensate: methodological recommendations and unresolved questions. Eur Respir J 2005; 26: $523-548$

2 Fireman E, Lerman Y. Induced sputum in occupational lung diseases. Review 2005; 2: 51 - 59

3 Obata H, Cittrick M, Chan Het al. Sputum eosinophils and exhaled nitric oxide during late asthmatic reaction in patients with western red cedar asthma. Eur Respir J 1999; 13: 489-495

${ }^{4}$ Lemiere C, Pelissier S, Tremblay Cet al. Leukotrienes and isocyanate-induced asthma: a pilot study leukotriene and isocyanat-asthma. Clin Exp Allerg 2004; 34: 1684-1689

5 Park HS, Jung KS, Hwang SCet al. Neutrophil infiltration and release of IL-8 in airway mucosa from subjects with grain dust-induced occupational asthma. Clin Exp Allergy 1998; 28: $724-730$

${ }^{6}$ Girand F, Chaboillez S, Cartier Aet al. An effective strategy for diagnosis occupational asthma use of induced sputum. Am J Respir Crit Care 2004; 170: 845 - 850

7 Baur X, Barbinova L. Latex allergen exposure increases exhaled nitric oxide in symptomatic healthcare workers. Eur Respir J 2005; 25: 309-316

8 Wild DM, Redlich CA, Paltiel AD. Surveillance for isocyanate asthma: a model based cost effectiveness analysis. Occup Environ Med 2005; 62: 743 - 749

9 Mapp CE, Boschetto P, Maestrelli Pet al. Occupational asthma. Am J Respir Crit Care Med 2005; 172: $280-305$

${ }^{10}$ Kraw M, Tarlo SM. Isocyanate medical surveillance: respiratory referrals from a foam manufacturing plant over a five year period. Am J Ind Med 1999; 35: 87-91

11 Tarlo SM, Banks DE, Liss Get al. Outcome determinants for isocyanate induced occupational asthma among compensation claimants. Occup Environ Med 1997; 54: 756 - 761

12 Tarlo SM, Liss GM. Diisocyanate-induced asthma: diagnosis, prognosis, and effectiveness of medical surveillance measure. Appl Occup Environ Hyg 2002; 17: 902 - 908

${ }^{13}$ Merget R, Caspari C, Dierkes-Globisch Aet al. Effectiveness of a medical surveillance program for the prevention of occupational asthma caused by platinum salts: a nested case-control study. J Allergy Clin Immunol 2003; 107: 707-712

${ }^{14}$ Smith TA. Preventing baker's asthma: an alternative strategy. Occup Med 2004; 54: 21 - 27

15 Jones KP, Rolf S, Stingl Cet al. Longitudinal study of sensitization to natural rubber latex among dental school students using powder-free gloves. Ann Occup Hyg 2004; 48: 455 - 457

${ }^{16}$ Tarlo SM, Liss GM. Can medical surveillance measures improve the outcome of occupational asthma? Allergy Clin Immunol 2001; 107: 583-585 
${ }^{17}$ Allmers H, Brehler R, Chen Zet al. Reduction of latex aeroallergens and latex-specific IgE antibodies in sensitized workers after removal of powdered natural rubber latex gloves in a hospital. J Allergy Clin Immunol 1998; 102: 841 - 846

${ }^{18}$ Hauptverband der gewerblichen Berufsgenossenschaften. Berufsgenossenschaftliche Grundsätze für arbeitsmedizinische Vorsorgeuntersuchungen. Stuttgart: Gentner, 2004

${ }^{19}$ Verordnung zum Schutz vor Gefahrstoffen (Gefahrstoffverordnung - GefStoffV) vom 23. Dezember 2004 (BGBl. I S. 3758), zuletzt geändert am 23. Dezember 2004 (BGBl. I S. 3855)

${ }^{20}$ LaMontagne AD. Cost effectiveness of surveillance for isocyanate asthma: finding an occupational health policy framework. Occup Environ Med 2005; 62: 741 - 742

${ }^{21}$ Palmberg L, Larsson B-M, Sundblad B-Met al. Partial protection by respirators on airways responses following exposure in a swine house. Am J Ind Med 2004; 46: 363 - 370

${ }^{22}$ Müller-Wening D, Neuhauss M. Protective effect of respiratory devices in farmers with occupational asthma. Eur Respir J 1998; 12: 569-572

${ }^{23}$ Gautrin D, Infante-Rivard C, Ghezzo Het al. Incidence and host determinants of probable occupational asthma in apprentices exposed to laboratory animals. Am J Respir Crit Care Med 2001; 163: 899-904

${ }^{24}$ Castro F. im Druck,

${ }^{25}$ Baur X, Preisser A. Asthma bronchiale and COPD. 2. Aufl. Stuttgart: Wissenschaftliche Verlagsgesellschaft, 2005

${ }^{26}$ Baur X(Hrsg). Lungenfunktionsprüfung und Allergiediagnostik. München-Deisenhofen: Dustri-Verlag, 1998

${ }^{27}$ Matthys H, Zaiss AW, Theissen JLet al. Definitionen, Soll- und Messwerte zur Diagnose obstruktiver, restriktiver sowie gemischter Ventilationsstörungen für die klinische Lungenfunktionsdiagnostik. Atemw-Lungenkrkh 1995; 21: 130-138

${ }^{28}$ Islam MS, Ulmer WT. Reference values in ventilatory lung function. Prax Klin Pneumol 1983; 37: $9-14$

${ }^{29}$ Ulmer WT, Gillissen A, Reichel Get al. Lungenfunktion und die Normwerte. Pneumologie 1993; 47: $403-408$

${ }^{30}$ Quanjer PH, Tammeling GJ, Cotes JEet al. Lung volumes and forced ventilatory flows. Report working party standardization of lung function tests. European Community for Steel and Coal. Official statement of the European Respiratory Society. J Eur Respir 1993; 6: 5-40

${ }^{31}$ Brändli O, Schindler C, Leuenberger PHet al. Reestimated equations for $5^{\text {th }}$ percentiles of lung function variables. Thorax 2000; 55: $173-174$

${ }^{32}$ Woitowitz HJ, Szadkowski D, Lehnert G. Feldstudie zum Normverhalten der arteriellen Blutgase und des pH berufstätiger Männer und Frauen vor und gegen Ende dosierter Belastung im Hinblick auf die Begutachtung. Archiv für Kreislaufforschung 1969a; 58: 35 - 53

33 Worth H, Breuer HWM. Deutsche Gesellschaft für Pneumologie - Empfehlungen zur Durchführung und Bewertung von Belastungsuntersuchungen in der Pneumologie. Pneumologie 1998; 52: $225-231$

${ }^{34}$ Eschenbacher WL. An algorithm for the interpretation of cardiopulmonary exercise tests. Chest 1990; 97: $263-267$

35 Padoan M, Pozzato V, Simoni Met al. Long-term follow-up of toluene diisocyanate-induced asthma. Eur Respir J 2003; 21: 637-640

${ }^{36}$ Vandenplas O, Toren K, Blanc PD. Health and socioeconomic impact of work-related asthma. Eur Respir J 2003; 22: 689-697

\section{Prof. Dr. med. Xaver Baur}

Ordinariat und Zentralinstitut für Arbeitsmedizin Hamburg

Seewartenstraße 10

20459 Hamburg

E-mail: baur@uke.uni-hamburg.de 
Welche Aussage zur Prävention arbeitsbedingter obstruktiver Atemwegserkrankungen trifft nicht zu?

Welche der folgenden Aussagen zur Therapie arbeitsbedingter obstruktiver Atemwegserkrankungen ist falsch?

\section{Welche Aussage trifft zu?}

Welche Aussage ist richtig?

Den begründeten Verdacht auf eine Berufskrankheit muss jeder Arzt per Formblatt melden und zwar an
A Unter Primärprävention versteht man die Elimination der gesundheitsgefährdenden Belastung am Arbeitsplatz

B Zur Primärprävention gehört das Tragen von persönlichen Schutzausrüstungen, z. B. Atemschutzmasken

C Primärprävention hat Vorrang vor der Sekundärprävention

D Zur Sekundärprävention gehören arbeitsmedizinische Vorsorgeuntersuchungen

E Im Rahmen der Tetiärprävention wird die Wiederherstellung der Gesundheit angestrebt

A Die medikamentöse Therapie unterscheidet sich nicht wesentlich von jener, die bei Atemwegserkrankungen anderer Genese durchgeführt wird

B Die Hyposensibilisierung ist nicht das Mittel der Wahl

C Die Karenz gegenüber der ursächlichen Noxe hat oberste Priorität

D Unter ausreichender Medikation und Vorsorgeuntersuchungen kann einer weiteren arbeitsbedingten krankheitsursächlichen Exposition (z. B. gegenüber Mehlstaub) zugestimmt werden

E Bei einer anerkannten Berufskrankheit trägt die Unfallversicherung die Behandlungskosten

A Die Prognose arbeitsbedingter obstruktiver Atemwegserkrankungen ist unter strikter Karenz gegenüber den ursächlichen Noxen stets gut

B Die Prognose arbeitsbedingter Atemwegserkrankungen ist unter strikter Karenz gegenüber den ursächlichen Noxen in der Mehrzahl der Fälle unbefriedigend

C Die Prognose ist unabhängig von der Dauer der unter Beschwerden weitergeführten Exposition

D Mittels einer regelmäßigen Vorsorgeuntersuchung lässt sich das Erkrankungsrisiko in der Regel nicht reduzieren

E Eine atopische Diathese stellt für alle Formen obstruktiver Atemwegserkrankungen ein hohes Risiko dar

A den Arbeitgeber

B die zuständige Fachkraft für Arbeitssicherheit im Betrieb

C die Krankenversicherung

D die zuständige Unfallversicherung und/oder die für den staatlichen Arbeitsschutz zuständige Stelle (staatlicher Gewerbearzt)

E es besteht kein Zwang einer Meldung 
Welche Aussage ist richtig?

Bei einer arbeitsbedingten richtunggebenden Verschlimmerung einer vorbestehenden obstruktiven Atemwegserkrankung durch Allergene oder Irritanzien am Arbeitsplatz

Welche Aussage ist falsch? Ein unspezifischer bronchialer Hyperreagibilitätstest
Welcher Test eignet sich nicht zum Nachweis einer Soforttyp-Sensibilisierung auf Berufsallergene?

Welche Sicherheitsvorkehrungen sind bei der Durchführung eines bronchialen Provokationstests nicht erforderlich?

Wie lange nach inhalativer Allergenprovokation ist der Patient zu überwachen?

Welche Antwort ist falsch?

Das Risiko, eine arbeitsbedingte obstruktive Atemwegserkrankung zu entwickeln, ist abhängig von
A besteht keine Berufskrankheit

B Ist der arbeitsbedingte Verschlimmerungsanteil als Berufskrankheit anzuerkennen und zu entschädigen

C ist das gesamte Krankheitsbild (also im vollen Umfang) als Berufskrankheit anzuerkennen und zu entschädigen

D kann man nur über die Öffnungsklausel (§9 Abs. 2 SGB VII), d. h. bei Vorliegen neuer wissenschaftlicher Erkenntnisse eine Berufskrankheit anerkennen

E ist in der Regel eine Entscheidung des Sozialgerichts erforderlich

A sollte bei Verdacht auf eine arbeitsbedingte obstruktive Atemwegserkrankung stets durchgeführt werden

B schließt bei negativem Ergebnis eine arbeitsbedingte obstruktive Atemwegserkrankung stets aus

C ist bei einer aktuellen Bronchialobstruktion kontraindiziert

D weist bei positivem Ergebnis auf ein Asthma bronchiale hin

E fällt häufig bei Rhinitis-Patienten positiv aus
A Epikutantest
D Pricktest
B Spezifische IgE-Antikörperbestimmung
E Intrakutantest
C Reibtest

A Das Personal muss für Notfälle ausgebildet und erfahren sein

B Während der Untersuchung muss jederzeit ein sachkundiger und in ErsteHilfe-Maßnahmen geschulter Arzt anwesend sein

C Die übliche Notfallmedikation muss verfügbar sein

D Vor Beginn muss im Untersuchungsraum ein inhalierbares kurz wirksames Bronchospasmolytikum zur sofortigen Anwendung bereit stehen

E Initial muss ein Plazebotest mittels Kochsalzlösung durchgeführt werden
A 30 Minuten
D 8 Stunden
B 2 Stunden
E 24 Stunden
C 4 Stunden

A der Höhe der Allergenbelastung am Arbeitsplatz

B der Höhe der Belastung durch Irritanzien

C genetischen Faktoren

D stets vom Rauchverhalten

E von vorbestehenden Atemwegserkrankungen 


\section{A. Angaben zur Person}

Online-Teilnahme unter http://cme.thieme.de

\section{B. Didaktisch-methodische Evaluation}

Name, Vorname, akad. Titel:

Straße, Hausnr.: PLZ/Ort:

Ich bin Mitglied der Ärztekammer:

Jahr meiner Approbation:

Ich befinde mich in der Weiterbildung zum:

Ich habe eine abgeschlossene Weiterbildung in/für:

seit/Jahr der Facharztanerkennung:

Spezialisierung innerhalb des Fachgebiets: $\square$ nein $\square$ ja, welche?

Ich möchte folgende Zusatzbezeichnungen erwerben:

Ich habe folgende Zusatzbezeichnungen: seit:

Ich bin tätig als:

$\square$ Assistenzarzt $\square$ Oberarzt $\square$ Chefarzt in folgender Klinik:

$\square$ Niedergelassener Arzt, seit $\square$ im Ballungsraum $\square$ im ländlichen Raum

$\checkmark$ Sonstiges (bitte eintragen):

Führen Sie in Ihrer Praxis diagnostische und therapeutische Auftragsleistungen im Bereich des Fortbildungsthemas durch?

$\square$ nein $\square$ ja, welche?

Bieten Sie in der Diagnostik und Therapie im Bereich des Fortbildungsthemas Besonderheiten in Ihrer Praxis/Klinik an?

$\square$ nein $\square$ ja, welche?

Machen Sie diese ggf. als Praxisbesonderheiten geltend?

$\square$ nein $\square$ ja, welche?

Ich bin Abonnent: $\square$ ja $\square$ nein, der Fragebogen ist aus/von: $\square$ Zeitschrift $\square$ thieme-connect $\square$ Kollegen $\square$ der Klinik $\square$ der Bibliothek $\square$ Sonstiges

1. Wie viele Patienten diagnostizieren/behandeln Sie im Zusammenhang mit dem Fortbildungsthema pro Jahr?

2. Bitte benennen Sie die 3 wichtigsten für Sie noch offenen Probleme in der Diagnostik des Fortbildungsthemas:

3. Bitte benennen Sie die 3 wichtigsten für Sie noch offenen Probleme in der Therapie des Fortbildungsthemas:

4. Bezüglich der Diagnostik/Behandlung im Rahmen des Fortbildungsthemas $\square$ fühle ich mich nach Studium des Beitrages in meiner Strategie bestätigt. $\square$ hat sich meine Strategie folgendermaßen verändert - bitte benennen: 


\begin{tabular}{l} 
Ihr Ergebnis \\
(wird vom Verlag ausgefüllt) \\
Sie haben_ von \\
richtig beantwortet und somit \\
$\square$ bestanden und 3 CME-Punkte \\
erworben \\
$\square$ nicht bestanden. \\
Stuttgart, \\
\hline (Stempel/Unterschrift) \\
zertifiziert durch die Ärztekammer \\
Nordrhein
\end{tabular}

C. Lernerfolgskontrolle (nur eine Antwort pro Frage ankreuzen)
5. Wurden aus der Sicht Ihrer täglichen Praxis wichtige Aspekte des Themas
a) außer Acht gelassen? $\square$ nein $\square$ ja, welche?
b) zu knapp abgehandelt? $\square$ nein $\square$ ja, welche?
c) überbewertet? $\square$ nein $\square$ ja, welche?

6. Etwa wieviel Prozent des Beitrages haben Ihnen

a) zur Auffrischung bereits bekannten Wissens gedient:

$a<10 \% \quad \square<25 \% \quad \square<50 \% \quad \square \geq 50 \%$

b) zur Erweiterung Ihres Spezialwissens gedient:

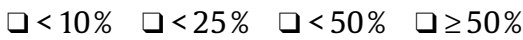

7. Die Diagnostik/Therapie im Zusammenhang mit dem Fortbildungsthema wird $\square$ von mir begonnen und vom Hausarzt weitergeführt.

$\square$ von mir begonnen und bis zur endgültigen Diagnosestellung bzw. Therapieeinstellung durchgeführt.

$\square$ Diagnostik, Therapie und Langzeitkontrolle erfolgen durch mich

(in Abstimmung mit dem Hausarzt).

$\square$ von mir überwiegend als Auftrags-/Konsiliarleistung erbracht.

8. Für die Diagnostik/Therapie zum Fortbildungsthema ziehe ich regelmäßig andere Fachgruppen hinzu. $\square$ nein $\square$ ja, welche?

9. Ergeben sich für Sie aus wirtschaftlichen Gründen Limitierungen im Einsatz von im Beitrag genannten Diagnose-/Therapieverfahren?

$\square$ nein $\square$ ja, welche?

10. Stehen Ihnen aus logistischen Gründen im Beitrag genannte Diagnose-/Therapieverfahren nicht/nur eingeschränkt zur Verfügung?

$\square$ nein $\square$ ja, welche?

11. Die Fragen lassen sich

$\square$ aus dem Studium des Beitrages allein beantworten.

$\square$ nur unter Zuhilfenahme zusätzlicher Literatur beantworten.

12. Ich habe für die Bearbeitung dieser Fragen (inkl. Antwortbogen) Minuten benötigt.

$\begin{array}{rrrrrrrrrrrr}\mathbf{1} & \text { A } & \text { B } & \text { C } & \text { D } & \text { E } & \mathbf{6} & \text { A } & \text { B } & \text { C } & \text { D } & \text { E } \\ \mathbf{2} & \text { A } & \text { B } & \text { C } & \text { D } & \text { E } & \mathbf{7} & \text { A } & \text { B } & \text { C } & \text { D } & \text { E } \\ \mathbf{3} & \text { A } & \text { B } & \text { C } & \text { D } & \text { E } & \mathbf{8} & \text { A } & \text { B } & \text { C } & \text { D } & \text { E } \\ \mathbf{4} & \text { A } & \text { B } & \text { C } & \text { D } & \text { E } & \mathbf{9} & \text { A } & \text { B } & \text { C } & \text { D } & \text { E } \\ \mathbf{5} & \text { A } & \text { B } & \text { C } & \text { D } & \text { E } & \mathbf{1 0} & \text { A } & \text { B } & \text { C } & \text { D } & \text { E }\end{array}$

\section{Erklärung}

Ich versichere, dass ich die Beantwortung der Fragen selbst und ohne fremde Hilfe durchgeführt habe.

Ort/Datum

Unterschrift

Nichtabonnenten bitte hier CME-Wertmarke aufkleben bzw. Abonnentennummer eintragen
Bitte senden Sie den vollständig ausgefüllten Antwortbogen und einen an Sie selbst adressierten und ausreichend frankierten Rückumschlag an den Georg Thieme Verlag KG, CME, Joachim Ortleb, Postfach 301120, 70451 Stuttgart. Einsendeschluss ist der 31. Mai 2007 (Datum des Poststempels). Die Zertifikate werden spätestens 14 Tage nach Erhalt des Antwortbogens versandt. Von telefonischen Anfragen bitten wir abzusehen. 
Für diese Fortbildungseinheit erhalten Sie 3 Fortbildungspunkte im Rahmen des Fortbildungszertifikates der Ärztekammern. Hierfür

- müssen 70\% Fragen richtig beantwortet sein.

- müssen die Fragen der Einheiten A bis D des Evaluationsbogens vollständig ausgefüllt sein. Unvollständig ausgefüllte Bogen können nicht berücksichtigt werden!

- muss Ihre Abonnentennummer im entsprechenden Feld des Antwortbogens angegeben oder eine CME-Wertmarke im dafür vorgesehenen Feld aufgeklebt sein.

\section{CME-Wertmarken}

CME-Wertmarken (für Teilnehmer, die die Zeitschrift Pneumologie nicht abonniert haben) können beim Verlag zu folgenden Bedingungen erworben werden: 6er-Pack Thieme-CME-Wertmarken, Preis 63,- Euro inkl. MwSt., Artikel-Nr. 901916; 12er-Pack Thieme-CME-Wertmarken, Preis 99,- Euro inkl. MwSt., Artikel-Nr. 901917. Bitte richten Sie Bestellungen an: Georg Thieme Verlag, Kundenservice, Postfach 301120, 70451 Stuttgart.

\section{Datenschutz}

Ihre Daten werden ausschließlich für die Bearbeitung dieser Fortbildungseinheit verwendet. Es erfolgt keine Speicherung der Ergebnisse über die für die Bearbeitung der Fortbildungseinheit notwendige Zeit hinaus. Die Daten werden nach Versand der Testate anonymisiert. Namens- und Adressangaben dienen nur dem Versand der Testate. Die Angaben zur Person dienen nur statistischen Zwecken und werden von den Adressangaben anonymisiert verarbeitet.

\section{Wichtige Hinweise}

Die CME-Beiträge der Pneumologie wurden durch die Landesärztekammer Nordrhein anerkannt. Die Zeitschrift für Pneumologie ist zur Vergabe der Fortbildungspunkte für diese Fortbildungseinheit berechtigt. Diese Fortbildungspunkte der Landesärztekammer Nordrhein werden von anderen zertifizierenden Ärztekammern anerkannt. Die Vergabe der Fortbildungspunkte ist nicht an ein Abonnement gekoppelt!

\section{Ausschneiden und schicken an: Georg Thieme Verlag, Kundenservice, Postfach 3011 20, 70451 Stuttgart Tel.: 0711/8931-333, Fax: 0711/8931-133, E-mail: Kundenservice@thieme.de}

Anschrift $\square$ privat $\square$ dienstlich
Titel, Name, Vorname:
Straße, Hausnr.:
PLZ/Ort:
ja, ich bestelle:
GThieme-CME-Wertmarken 6er-Pack, Preis 63,- Euro inkl. MwSt. und Versand,
Artikel-Nr. 901916
aThieme-CME-Wertmarken 12er-Pack, Preis 99,- Euro inkl. MwSt. und Versand,
Artikel-Nr. 901917
Ort/Datum:
Unterschrift/Stempel:
Preisänderungen und Irrtümer vorbehalten!

